\title{
Coronary risk reduction intervention for siblings and offspring of patients with premature coronary heart disease: the CRISO study protocol for a randomised controlled pilot study
}

\author{
Justin Lee Mifsud ${ }^{1,2^{*}}$ (D) John Stephenson ${ }^{3}$, Felicity Astin ${ }^{3,4}$ and Joseph Galea ${ }^{2}$
}

\begin{abstract}
Background: Research has consistently demonstrated that preventive cardiology programs have limited success, and healthy practices among high-risk individuals remain suboptimal. Furthermore, there are no current programmes in Malta that offer support to first-degree relatives of patients with premature coronary heart disease. This internal pilot study will determine the feasibility, acceptability, and potential effectiveness of a preventative intervention.

Methods/design: We are conducting a 12-month single-centre, two-armed group randomised controlled trial $(R C T)$, recruiting a sample of 100 asymptomatic first-degree relatives of patients with premature coronary heart disease (CHD). The study seeks to test an evidence-based intervention to reduce modifiable risk and determine its feasibility and acceptability. The Intervention will be delivered at an outpatient office based in a large acute academic hospital. It will comprise risk communication using an online risk calculator, a counselling style adapted from motivational interviewing, and 12 weekly telephone goal reinforcement calls (3 months). Control subjects will receive verbal lifestyle advice only. Feasibility will be assessed through recruitment and retention. Qualitative evaluation interviews will be conducted with a subsample of 24 purposefully selected participants at 12 months. Assessment for risk factor changes will be measured at pre-intervention and 6 and 12 months. Associations between variables will also be assessed descriptively.

Discussion: Preventive cardiology guidelines highlighted the importance of lifestyle interventions, and lifestyle intervention adherence was proven to reduce atherosclerotic cardiovascular disease (ASCVD) risk, regardless of the individual's genetic risk. Preventive cardiology programmes may fail to adequately support persons in modifying risky behaviours, and research demonstrates that healthy practices among high-risk individuals can remain suboptimal.

Siblings and offspring of patients with premature CHD are at increased risk of ASCVD. Despite this, there is no process in place for routine screening and support to modify risk. It is hypothesised that participants assigned to
\end{abstract}

\footnotetext{
* Correspondence: justin-lee.mifsud@um.edu.mt

${ }^{1}$ Faculty of Health Sciences, University of Malta, Msida, Malta

${ }^{2}$ Faculty of Medicine and Surgery, University of Malta, Msida, Malta

Full list of author information is available at the end of the article
}

(C) The Author(s). 2021 Open Access This article is licensed under a Creative Commons Attribution 4.0 International License, which permits use, sharing, adaptation, distribution and reproduction in any medium or format, as long as you give appropriate credit to the original author(s) and the source, provide a link to the Creative Commons licence, and indicate if changes were made. The images or other third party material in this article are included in the article's Creative Commons licence, unless indicated otherwise in a credit line to the material. If material is not included in the article's Creative Commons licence and your intended use is not permitted by statutory regulation or exceeds the permitted use, you will need to obtain permission directly from the copyright holder. To view a copy of this licence, visit http://creativecommons.org/licenses/by/4.0/ The Creative Commons Public Domain Dedication waiver (http://creativecommons.org/publicdomain/zero/1.0/) applies to the data made available in this article, unless otherwise stated in a credit line to the data. 
the intervention arm will show more cardio-protective lifestyle-related improvement from the baseline than those
in the control group. To date, this is the first trial being conducted amongst Maltese first-degree relatives. This study
addresses the needed research, and the results will inform a definitive trial.
The funding institution is the University of Malta.

Trial registration: ISRCTN, ISRCTN21559170; Registered 06/08/2020,

Keywords: Cardiovascular disease, Prevention, Modifiable risk, Feasibility, Acceptability, Protocol, Pilot

\section{Background and rationale}

An important risk factor for atherosclerotic cardiovascular disease (ASCVD) development is family history [1]. A subset of ASCVD is coronary heart disease (CHD). The risk of having a CHD event is increased in the families of affected patients who had a premature atherosclerotic event [2], defined as an event occurring in males before 55 years and before 65 years in females [3]. In Malta, about $40 \%$ of cardiac patients will present with premature CHD [4]. First-degree relatives, such as siblings of patients with premature CHD, have an increased risk of developing the disease themselves by approximately $40 \%$, while offspring of patients with premature CHD have about a $60 \%$ to $75 \%$ increased risk when compared with the general population [5]. Moreover, the risk is higher in males than in females $[6,7]$ and more substantial in middle-aged persons [8]; it increases with the number of affected relatives $[5,9]$ and even more so if the diseased vessel is a main left coronary artery [10].

Siblings and offspring of CHD patients are also more likely to have a higher prevalence of central obesity $(\geq 102 \mathrm{~cm}$ in Europid men; $\geq 88 \mathrm{~cm}$ in Europid women), hypertension, hypercholesterolemia, and smoking than people who do not have a first-degree relative with CHD [11-14]. In turn, these outcomes can concurrently act as determinants for subsequent CHD events [3, 15]; however, they can be modified or controlled. Preventive cardiology guidelines highlighted the importance of lifestyle interventions $[3,16]$. Research shows that smoking cessation [17-20], adherence to a Mediterranean diet, and physical activities reduce ASCVD incidence and mortality [21-23]. Also, adherence to a Mediterranean diet and physical activity reduce risk regardless of the individual's genetic risk [24-28]. However, a gap between scientific evidence and clinical practice exists, and current prevention programmes do not support individuals with a strong family history in identifying and modifying their risk. Primary prevention is essential among individuals at increased risk $[3,16]$.

A recent systematic review with a meta-analysis of preventive programmes highlighted the application of counselling elements to help individuals improve their heart health risk [29]. These identified elements included: compassion, listening, affirmation, evocation, use of open questions, summarising and resolving ambivalence. Such elements could be combined with educational resources. Barrier change identification and goal setting were also identified as essential elements. It was also suggested that the application of Motivational Interviewing (MI) communication skills; open-ended questions, affirmation, reflection and summarisation (OARS), could be combined with the application of ASCVD risk scores [29]. Although a recent meta-analysis using risk scores in primary prevention efforts showed no evidence in reducing ASCVD event outcomes, this could be explained by the low event rate in individuals without preexistent ASCVD. Therefore, it may not be feasible or practical to conduct a large trial to evaluate ASCVD risk calculators' effectiveness in reducing ASCVD outcomes [30]. However, it would be more practical to focus on modifiable risk factor change or adherence to lifestyle therapies. It is necessary to conduct further research to evaluate factors that enable and motivate individuals at risk, yet without symptomatic pre-existent ASCVD, to support them in adopting healthy lifestyle changes [29, 31].

This approach could be ideal amongst first-degree relatives of patients with premature ASCVD. Risk factor evaluation, education, and communication using counselling elements and risk scores may help establish a successful cardiovascular risk factor change.

\section{Aim and objectives}

This study aims to evaluate a pilot study of a preventive cardiology program designed to promote risk reduction in a sample of asymptomatic first-degree relatives of patients with premature CHD.

Primary objectives are as follows:

1. Feasibility: To assess the recruitment processes and study uptake to inform the feasibility of a largescale randomised controlled trial

2. Acceptability: To determine whether the Intervention for risk reduction is acceptable by first-degree relatives

Secondary objectives are as follows: 
3. Potential effectiveness: To determine if the programme provides preliminary evidence for modifying lifestyle behaviours; physiological, anthropometric, biochemical parameters; Heart Age (estimated age based on ASCVD risk); and health literacy

4. Association between variables: To determine if the programme provides preliminary evidence for an association with lifestyle factors such as Mediterranean diet and physical activity, which themselves can act as determinants to clinical outcomes such as BMI/WC/lipids/blood pressure/ HbA1c.

\section{Methods/design}

\section{Setting}

ASCVD risk evaluation, delivery of Intervention, and outcome measurements are being carried out from an outpatient clinic based at the Malta General State Academic Hospital.

\section{Study type and design}

The study is an interventional pilot study. The design consists of a single-centre, 2-group pilot trial consisting of a target sample of 100 first-degree relatives. This design was chosen to determine the association with and effectiveness in addressing the modifiable cardiovascular risk factors. Qualitative evaluation interviews will be conducted with a subsample of 24 purposefully selected participants at 1 year. In this way, quantitative and qualitative data strengthen the study, allowing it to achieve all its proposed objectives [32]. More information about the study is available on a registry platform (http://www. isrctn.com/ISRCTN21559170).

\section{Materials}

\section{Sociodemographic questionnaire}

A questionnaire was designed to collect sociodemographic data, including questions on age, sex, educational level, employment status, time of the patient coronary event, and whether participants live alone or live with others. These variables will be collected to characterise the sample. However, as randomisation is not guaranteed to result in perfectly balanced groups at baseline, variables that may be associated with the outcome will be checked for balance and controlled for in subsequent analysis if necessary.

\section{Primary outcomes Feasibility}

Feasibility will be ascertained by evaluating the recruitment and intervention processes. The study's feasibility requires that the Intervention be effectively carried out amongst an adequate number of first-degree relatives over 2 years. The assessment of the feasibility of the recruitment process will include records of the number of first-degree relatives:

- Reached via patients/video clip (link to video clip)/ poster (link to poster)/ study participants/ clinician,

- Who expressed interest in the study,

- Who are potentially eligible, with reasons for ineligibility,

- Who agreed to participate in the trial,

- Lost to follow-up (retention and drop-out rate),

Furthermore, the assessment of the feasibility of the Intervention will include the time taken to:

- Complete the cardiovascular risk assessment,

- Deliver the Intervention (in-person session and telephone sessions).

\section{Acceptability}

Acceptability will be evaluated by determining how well the Intervention is received by first-degree relatives and the extent to which this Intervention could meet their needs. Post-intervention questionnaires and interviews will be used to collect this data. This requires data of the participants' most valued intervention components in terms of being supportive and the participants' views of the quality of interactions during intervention sessions, along with how well the intervention targets can be incorporated into participants' lifestyles, taking into consideration any difficulties participants might have experienced while attempting to comply with the programme goals. We will evaluate the internal reliability (i.e. internal consistency) of self-produced questionnaires during the piloting process using Cronbach's alpha scores to identify any items that respondents are not answering consistently with their responses to other items before general implementation. We will calculate the alpha reliability value for the whole scale and will also infer individual item reliability via alpha values calculated for the scale with each item deleted in turn. Any items identified as detracting from overall reliability will be considered for removal or re-wording and re-piloted if necessary until satisfactory scale reliability is achieved.

\section{Secondary outcomes \\ Potential intervention effectiveness}

Preliminary evidence for the effect of the intervention will be determined by an increase in the mean smoking cessation attempts, increased mean Mediterranean diet score, and physical activity level. A decrease in the mean serum lipids, blood pressure level, waist circumference, and body mass index, and Heart Age will be considered as improvements in cardiovascular risk factors if 
statistically significant. Any trends identified across variable results will be explored.

The following are secondary outcome measurements considered:

\section{- Smoking status}

Smoking status will be answered by a 'yes' or 'no.' As self-reporting depends on participants' honesty, those who report that they have stopped smoking will be tested for expired carbon monoxide using a carbon monoxide monitor.

\section{- Mediterranean diet score}

The 14-point Mediterranean Diet Adherence Screener (MEDAS) [33] is a fourteen-item questionnaire that assesses adherence to the Mediterranean diet with two 'yes-or-no' questions on food intake habits and another twelve questions on food consumption frequency. The questionnaire is based on the nine-item index used in the PREDIMED study, and it covers the use of Mediterranean diet habits and frequency of consumption of Mediterranean food items. If the item/question is not met, a score of zero will be recorded. Therefore, the final score will range from 0 to 14 . The higher the score, the greater the adherence to the Mediterranean diet.

\section{- Physical activity level}

The rapid assessment of physical activity (RAPA) questionnaire is a 9-item valid questionnaire, which requires 2 to $5 \mathrm{~min}$ to complete [34]. The questionnaire is based on the Centres for Disease Control and Prevention (CDC) guidelines, suggesting a minimum of $30 \mathrm{~min}$ of moderate physical activity daily. The questionnaire covers a range of physical activity levels, from sedentary to regular vigorous physical activity and strength training and flexibility. The total score of the first seven items is one score for each and, thus, scoring is from 0 to 7 points. Responses to strength training and flexibility items are scored separately, with strength training $=1$ point, flexibility $=2$ points, or both $=3$ points. Therefore, the final score would range from 0 to 10 .

\section{- Biochemical measures}

Blood biochemicals (lipids, HbA1c) will be analysed using the Roche COBAS analyser, while TFT will be analysed using the Siemens ADVIA Centaur analyser. Both analysers are closed systems, and the kits that will be used are only those provided by Roche and Siemens, respectively.

- Physiological measures
Resting physiological measurements will be measured using an Omron ${ }^{\mathrm{Tm}}$ blood pressure monitor, measured two to three times by auscultation, and an average will be taken as the final measurement. Heart rate shall be measured manually using the radial pulse. For both measurements, the first measurement shall be monitored after a 10 -min seated rest, repeated $10 \mathrm{~min}$ after, and then again at the end of the session. Blood pressure will be measured in both arms.

\section{- Anthropometric measures}

Anthropometric measurements will be measured using a SECA scale with a height gauge. Numerous steps will be taken to avoid measurement errors. Weight will be taken to the nearest $0.1 \mathrm{~kg}$ and both measurements recorded twice. Recording of the waist circumference will be recorded while the participant is standing. A nonelastic measuring tape will be placed at the midpoint between the iliac crest and the lowest rib and at the umbilicus level. This measurement will be taken at the end of expiration.

\section{- Heart Age}

Heart Age will be calculated after obtaining the following parameters: age, sex, systolic blood pressure, cholesterol, smoking status, weight, and height. The parameter data obtained will be inputted into the online calculator to generate a score. This score will allow for the observation of changes in global risk [35].

\section{- Health literacy}

The Health Literacy Questionnaire (HLQ) has nine scales in total $[36,37]$. We decided to select those scales which we think are relevant to the Intervention. The questions will not be amended as they are validated in the way they are. However, the researcher shall tell the participant that the questions asked are about their heart health.

The scales selected are: scale 1, Feeling understood and supported by healthcare professionals; scale 2, Having sufficient information to manage health; scale 3, Actively managing health.

The selected scales' response options range from 1 to 4 , with the scoring as follows: 'strongly disagree' $=1$, 'disagree' $=2$, 'agree' $=3$, 'strongly agree' $=4$. The total score is calculated for all the questions on that scale and divided by the number of items.

\section{Participants}

The study population will consist of first-degree relatives of patients with premature CHD (males $\leq 55$, females $\leq$ 
65), aged 30 years and older. Males and females of Maltese ethnicity seeking cardiovascular risk assessment with no history of cardiovascular disease will be recruited for the study. Other eligibility criteria also apply:

- Participants must not have diabetes.

- Participants must not have a history of rheumatoid arthritis and chronic kidney disease.

- Participants must not have contraindications against physical activities and can go up a flight of stairs comfortably.

- Participants must not be pregnant.

\section{Clinician to deliver the Intervention}

The clinician to deliver the Intervention is a qualified nurse who received training from Imperial College London in preventive cardiology (JLM). The clinician has also received training in MI from Sheffield Hallam University. Additionally, the clinician has over 8 years of clinical experience working in the cardiology department.

\section{Sampling frame}

About seven acute coronary syndrome (ACS) patients are admitted to the acute general hospital per week, including unstable angina, non-ST and ST-segment elevation ACS. About $40 \%$ will present with premature ACS [4]. Maltese individuals who are 30 years and older often have quite extensive families. Across 52 weeks (1 year), logistically, if every patient informs their relatives, this would amount to over 100 eligible participants. Since there are no records concerning the recruitment uptake and attrition rate, we will dedicate two years for recruitment and data collection. A sample of 100 participants should be adequate to prove that the process is viable. As the pilot study is not expected to be powered to detect differences between groups, there is no universally accepted calculation for the pilot study sample size [38]. If a meaningful group difference is unknown and the pilot study is intended to establish an effect size for sample size calculation, it is recommended to have 30 to 40 participants recruited in each group. Recommendations for feasibility studies propose a minimum of 30 participants per arm to estimate parameters for future sample size calculations $[39,40]$. Ultimately, the sample size decision for a pilot RCT must also consider the research timeline, human resources and costs, and the research objectives [40, 41].

\section{Procedures}

\section{Recruitment}

The flowchart displayed in Fig. 1 summarises the study design and participant flow, from enrolment to the final follow-up. Before discharge, all patients with acute coronary syndrome are enrolled in a hospital-based cardiac rehabilitation program. Patients with premature CHD, who attend cardiac rehabilitation, will be informed by their nurse about the study, which concerns their relatives. Patients will be the source of help to recruit study participants. Poster promotion of the study will be available at the cardiac rehabilitation reception area and local pharmacies. Poster and video clip promoting the study will be shared via the University's social media (Facebook, News point). Those having a sibling or a parent with premature $\mathrm{CHD}$ and are seeking cardiovascular risk assessment will be asked to answer a few questions via telephone to assess if they meet the inclusion criteria. Those found to be eligible will be invited for their baseline assessment at the hospital outpatient clinic.

\section{Recruitment method for interviews}

All the intervention participants will be asked to complete a questionnaire at the end of the programme.

From a sample of first-degree relatives who completed the programme, 24 individuals will be asked to participate in the interviews. A stratified purposeful sample will be chosen varying on preselected parameters deemed analytically relevant to the Intervention, providing maximum variation. These are sex, age, and risk profile at 12 months. Participants for whom post-intervention outcomes did not improve and participants who had improved their risk profile at 12 months will be purposively selected for focused analysis to identify the Intervention's strengths and weaknesses [32].

\section{Group randomisation}

Before the baseline/pre-intervention evaluation, approximately 100 participants shall be randomly allocated to one of the two groups:

1. The intervention group shall consist of 50 participants. Participants in this group will have one face-to-face session (evaluation of modifiable risk factors, 2-way risk factor communication, education, and individualised counselling to set up a cardio-protective documented plan) followed by weekly telephone-based cardio-protective reinforcement sessions for up to 12 weeks.

2. The control group shall consist of 50 participants. Participants will have one face-to-face session of verbal lifestyle advice only.

Block randomisation will be applied to balance out the sample size between the intervention and control groups. An opaque sealed envelope technique, using block randomisation with blocks of size four, will be implemented for this study. This method will allow an equal chance of being allocated to one of the two groups 

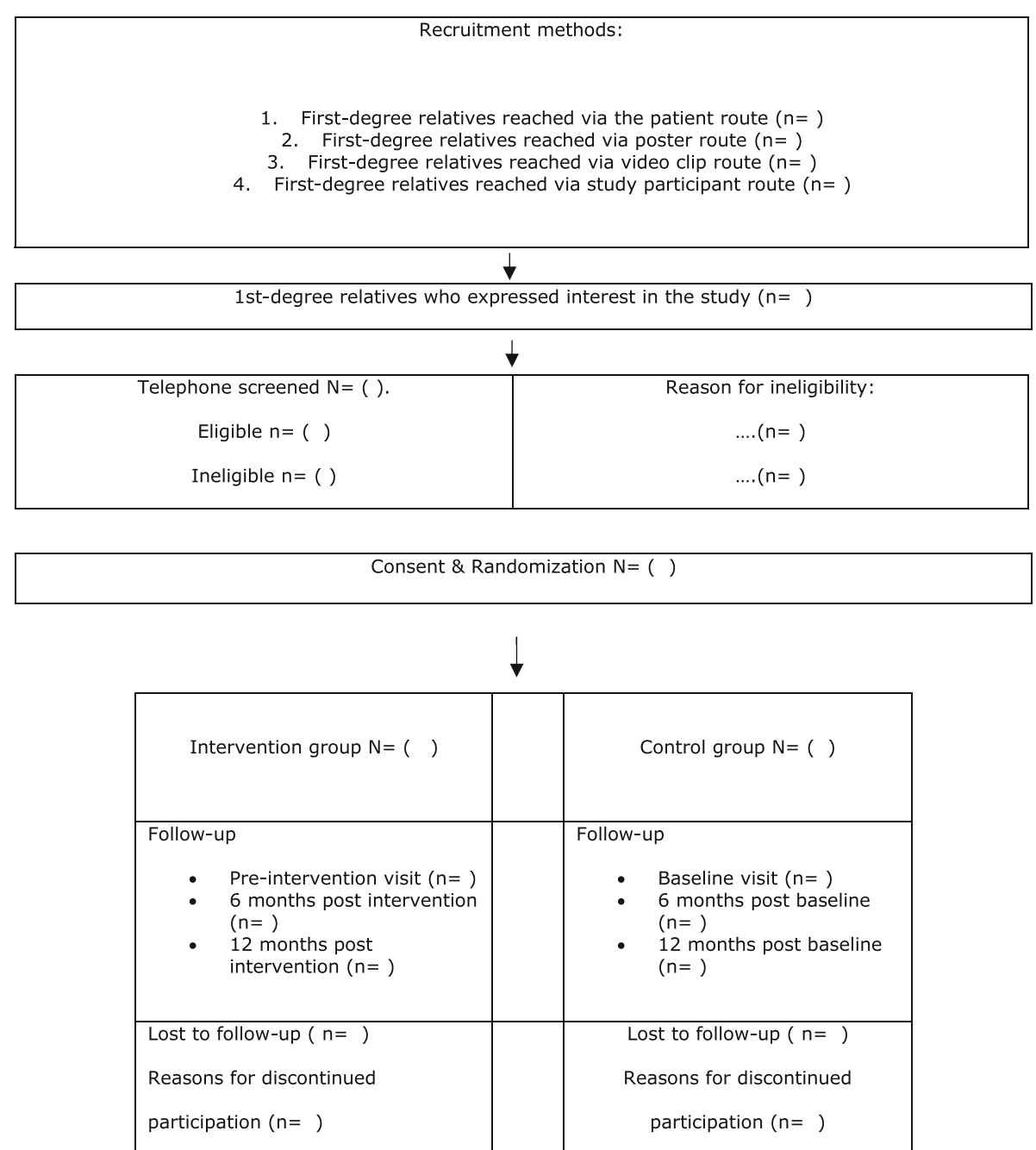
i. Post-intervention questionnaire (intervention group) 12 months post-
pre-intervention visit

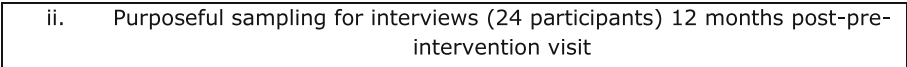

Fig. 1 Spirit (Standard Protocol Items: Recommendations for Interventional Trials) flow diagram

and ensure that near-equal numbers are assigned to each group, minimising the risk of group imbalances and allocation bias.

\section{Blinding and allocation concealment}

Awareness of being part of the intervention group may result in participants behaving differently to what they otherwise might do. Group awareness could have implications for the study's internal and external validity. The participants were informed about the aim of the study; however, not about to which group they have been allocated. Furthermore, participants will be allocated by an independent person from the study, and allocation will occur before the baseline/pre-intervention risk evaluation. Due to resource limitations, the study investigator will deliver the Intervention and assess the outcomes, therefore making part of the blinding impossible. However, blinding will be imposed on the data analyst.

\section{Intervention and control arm}

All participants will receive the intervention/control in 2-3 days of completing their baseline/pre-intervention assessment. This period will allow time for blood 
investigation results to be reported on the electronic health records.

Intervention arm Table 1 shows the intervention content and the behaviour change techniques (BCTs) [42] used in the CRISO intervention. Delivery of the CRISO intervention is personalised to the individuals' specific needs. It allows for flexibility across different participants to maximise support in improving their lifestyle behaviours. This would be achieved through modifiable risk factor evaluation, personalised risk communication, and education using Heart Age. A counselling style approach that draws upon some but not all MI principles and practices will be applied. The participant will be asked to reflect and elaborate on their risk profile. This will include the identification and selection of risk factors that the participant would like to modify. A target goal with specific actions to accomplish will be developed with the clinician's support. Figure 2 provides a central illustration of the program. The algorithm will support firstdegree relatives more systematically and equip them with a set of specific actions that they can take to reach the target goal. Table 2 gives a greater focus on the use of specific actions. The clinician will also ask the participant to identify a person who can motivate them to implement specific actions. Reflection on obstacles to change and ways to overcome such challenges are also taken into consideration. Summarising will occur at the end of the session, where the clinician will highlight the target goal and the specific actions selected to reach the goal. This will demonstrate that the clinician was actively listening and listening to understand and not to respond. The clinician will support the participant's strengths to increase their confidence in making change. At the end of the session, an electronic preventive cardiology report (Table 3) will be forwarded to the participant. The report will include a cardioprotective plan developed by the participant and the clinician through discussion. The report is to be updated for the participant at 6 months and 12 months follow-up (Table 3).

Telephone reinforcement sessions following the combined risk communication and counselling session will also be used. In total, participants receive one in-person session and 12 telephone calls (occurring between weeks 1 and 12).

Theoretical model The chosen theoretical model of behaviour change is the Capability Opportunity Motivation-Behaviour model (COM-B). Other theoretical frameworks, such as the behavioural choice theory or theory of planned behaviour, social cognitive theory, and self-regulation theory, appeared ineffective in similar studies [29]. The behaviour change wheel is a theoretical framework that led to the development of the COM-B model. The COM-B is a simple, cost-effective model to apply to all behaviours [43-45]. The model is pivotal in simplifying how behaviours could be targeted [46] and aids in supporting change in one or more of the following: capability, opportunity, and motivation relating to the behaviour itself or behaviours that compete with or support it. Drafting the programme using the COM-B model allowed for insights into how the programme intervention could alter risky behaviours. The COM-B model theory states that there must be the 'capability' for modification to modify behaviour (sedentary lifestyle, unhealthy dietary eating patterns, and smoking). Firstdegree relatives must have the physical and psychological capability to modify risk by developing knowledge through professional contact, discussing risk via visual aids, and discussing risk-reduction methods through the provision of feedback and monitoring. The CRISO program may facilitate an environment for opportunistic screening, improve professional contact, and encourage first-degree relatives to seek risk assessment. The Heart Age risk calculator was identified to educate about, evaluate, and communicate risk to increase understanding of risk and support participants to act on it. In return, this should improve understanding of risk and enhance the capability and opportunity to modify risk factors. Using a counselling style adapted from MI might further support individuals in identifying and select behaviours that require modification [29].

Control arm About 70\% of general practitioners' CVD risk consultations happen in verbal terminology [47]; participants assigned to the control arm will only receive one in-person session based on verbal lifestyle advice, referring to the European Society of Cardiology lifestyle targets about smoking, dietary pattern, and physical activity levels [3].

\section{Follow-up}

Participants will be assessed at the same time points after being recruited in the study. Follow-up points for study participants translate into the following phases: baseline/pre-intervention, 6 months, and 12 months after baseline/pre-intervention. Table 4 shows data collection points.

\section{Data analysis}

\section{Descriptive summary of sample data}

A description of participants' characteristics, including demographic information and baseline measurements, will be presented for both groups.

The entire cohort will also be summarised descriptively. For categorical data (smoking), frequencies and valid proportions and percentages will be used [48]. $\mathrm{Nu}$ merical variables will be summarised using mean and 
Table 1 Content summary for the CRISO intervention and BCTS

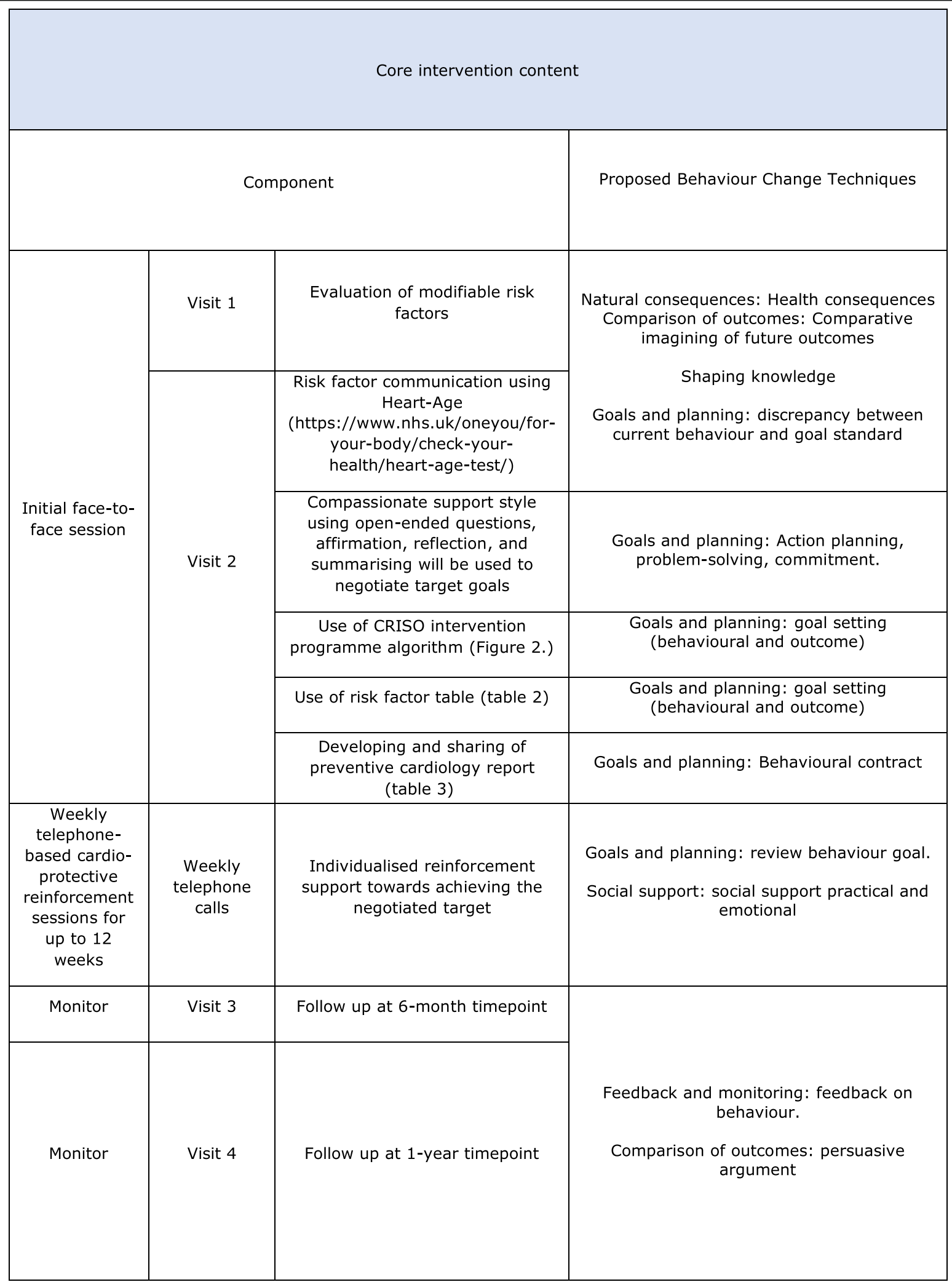




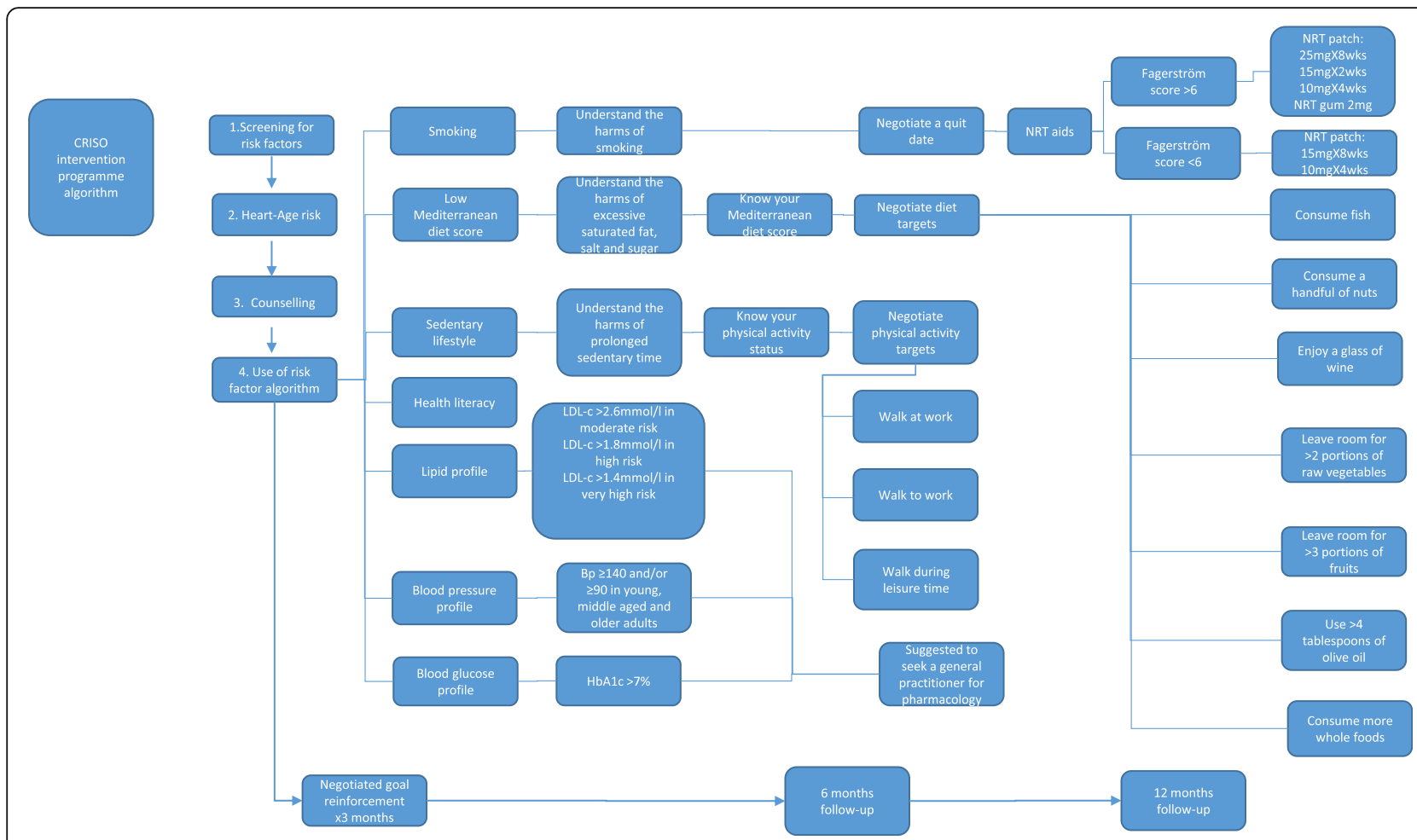

Fig. 2 CRISO intervention programme algorithm

standard deviation, or median and range or interquartile range. Risk ratios will be generated for selected outcome variables, dichotomised as necessary, from proportions of participants meeting particular criteria (e.g. control of blood pressure) in the control and intervention groups.

\section{Primary outcome analysis (Quantitative and Qualitative data)}

Objective 1: Feasibility The proportion of those who showed interest in the study and are eligible to participate, agreed to participate, and those who remained in the study will be recorded. Percentages (\%) of the number approached for participation will be used [48]. The average time to deliver the Intervention will be measured.

Objective 2: Acceptability A post-intervention questionnaire, consisting of ruler scales $(0-10)$, was developed to rank the intervention components' order. This data will be collected from all the intervention participants.

The audio-recorded data from the interviews shall be transcribed verbatim by the author. The data will be validated with the study participants and analysed using a thematic analysis method [49]. The phases to be applied are familiarisation with the data and identifying items of interest, code generation, management of the coding process, reviewing potential themes, defining and labelling the themes, and producing a report [49].

\section{Secondary outcome analysis}

Objective 3: Estimates of effect and preliminary evidence of efficacy The likely range of effects will be estimated using liberal confidence intervals. Preliminary estimates of intervention efficacy will be established via hypothesis tests with liberal alpha values (e.g. 0.15, 0.2). Numerical outcomes measured on an interval level, and numerical outcomes measured on an ordinal scale that can be considered approximate to interval level, will be assessed using independent samples $t$ tests. Categorical data will be assessed using the chi-squared test for association.

Intention-to-treat analysis will be used to test for the differences between the two groups at the endpoint [50]. Intention-to-treat analysis is considered the gold standard method for evaluating a new intervention. This method includes all study participants whether or not they have completed the study program and will preserve the randomisation process as it keeps the groups balanced in their number of participants in case of any drop-outs, thus minimising biases [51].

Risk ratios (RRs) will be generated for selected outcome variables, dichotomised as necessary, from 
Table 2 Intervention risk factor and behavioural targets

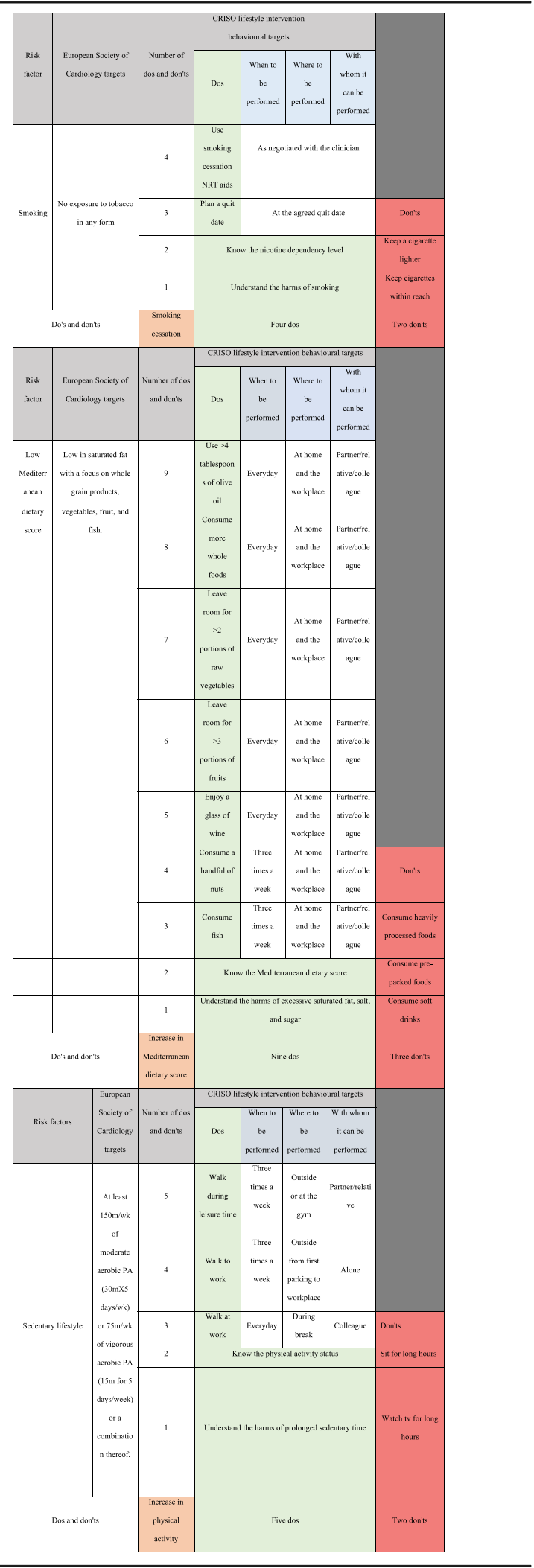

proportions of participants meeting particular criteria (e.g. control of blood pressure) in the control and intervention groups.

Effect sizes such as Cohen's d statistics will be calculated for each outcome measure and presented alongside parameter estimates in each group and associated confidence intervals. These, in conjunction with other statistics derived from the pilot analysis, may be used to estimate sample size for a subsequent follow-up RCT [38].

Objective 4: Preliminary indications of associations The preliminary indications of associations between outcome variables will be facilitated using correlation and association measures (Pearson's product-moment correlation coefficient for numerical outcomes) (uncorrected chi-squared test for association and/or Fisher's exact test for categorical outcomes). Liberal confidence intervals (e.g. 80\%, 90\%) will be applied.

\section{Ethics}

A detailed information letter will be provided to each participant. This letter will explain the nature of the study and what participation would involve. After the participant is given time to clarify anything about study participation, the principal researcher will obtain each participant's consent. It will be made clear that data will be retained in an anonymous form. Participants are free to withdraw at any time during the study. Data will be safely stored in an encrypted computer, and only the principal researcher will have access to this data. This is per data protection directives (GDPR EU 2016/679). The protocol was approved by the University of Malta Ethics Committee (UNIQUE FORM ID: 3756_191119) and the Malta Health Ethics Committee (HEC03 CT01/20).

\section{Discussion}

This study aims to evaluate a pilot study of a preventive cardiology program designed to promote risk reduction in a potential risk group. Issues of feasibility in the study design need to be tested before conducting a larger-scale randomised controlled trial [52]. The testing requires determining acceptability, estimating participants' recruitment and retention rate, evaluating the Intervention's operational strategy, and estimating the sample size needed for a full-scale study. The Medical Research Council framework guidance reports that a pilot study must be a scale model of the planned full-scale Intervention and must identify and address any issues [52].

Having access to a cardiovascular risk assessment (Opportunity) and being able to understand heart risk and ways to change risk (Capability) might increase motivation to modify present risks (Fig. 3). The key determinant is motivation; however, motivation alone is not 


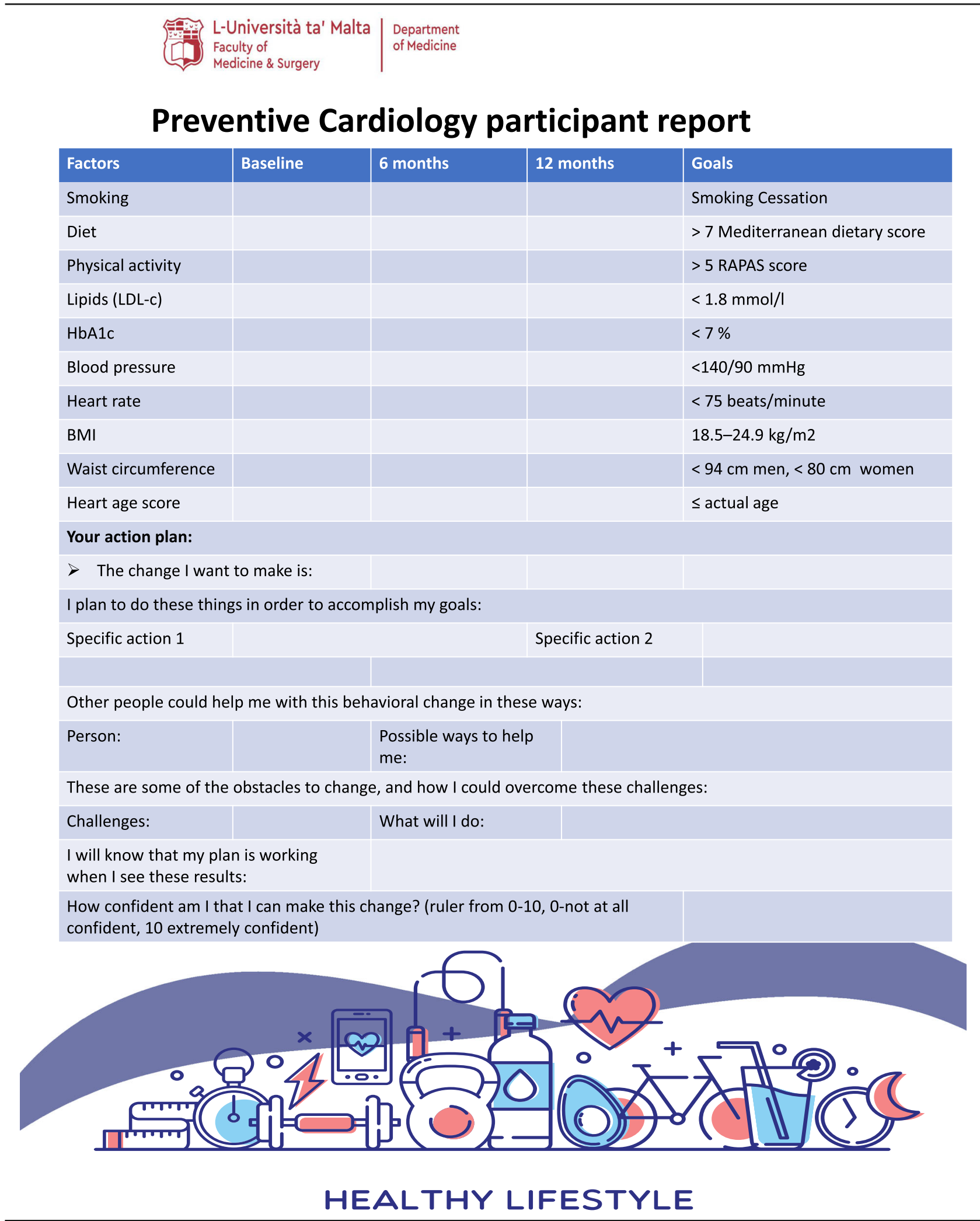


Table 4 Data collection points

\begin{tabular}{|c|c|c|c|c|}
\hline \multicolumn{5}{|c|}{ STUDY PERIOD } \\
\hline & Enrolment & Allocation & Post-allocation & Close-out \\
\hline TIMEPOINT & $-I_{1}$ & $I_{0}$ & $I_{1}$ & $\underline{T}_{z}$ \\
\hline ENROLMENT: & & & & \\
\hline Telephone eligibility screening & $\underline{\square}$ & & & \\
\hline $\begin{array}{l}\frac{\text { Informed consent on the first }}{\text { visit }}\end{array}$ & $\underline{\sim}$ & & & \\
\hline Allocation & & $\underline{v}$ & & \\
\hline INTERVENTIONS: & & & & \\
\hline [Intervention] & & $\underline{\underline{v *}}$ & & \\
\hline [Limited Intervention] & & $\underline{v * *}$ & & \\
\hline Measure(s) & & Baseline/pre-intervention & 6-month time point & 1-year time point \\
\hline & & $\underline{T_{0}}$ & $\underline{T_{1}}$ & $\underline{T_{2}}$ \\
\hline Sociodemographic & & $\underline{v}$ & & \\
\hline Feasibility & $\underline{\sim}$ & & & $\underline{\sim}$ \\
\hline Acceptability & & & & $\underline{v}$ \\
\hline Smoking status & & $\underline{v}$ & $\underline{v}$ & $\underline{\sim}$ \\
\hline Mediterranean dietary score & & $\underline{v}$ & $\underline{v}$ & $\underline{v}$ \\
\hline Physical activity level & & $\underline{v}$ & $\underline{\sim}$ & $\underline{v}$ \\
\hline Biochemistry measures- Lipids & & $\underline{v}$ & $\underline{v}$ & $\underline{\sim}$ \\
\hline $\begin{array}{c}\text { Biochemistry measures- } \\
\text { HbA1C }\end{array}$ & & $\underline{v}$ & $\underline{v}$ & $\underline{v}$ \\
\hline Physiological measures & & $\underline{v}$ & $\underline{\sim}$ & $\underline{v}$ \\
\hline Anthropometric measures & & $\underline{v}$ & $\underline{v}$ & $\underline{v}$ \\
\hline Heart age score & & $\underline{v}$ & $\underline{v}$ & $\underline{\sim}$ \\
\hline Health literacy & & $\underline{\sim}$ & $\underline{v}$ & $\underline{v}$ \\
\hline Anti-hypertensive drug use & & $\underline{v}$ & $\underline{v}$ & $\underline{\sim}$ \\
\hline Anti-diabetic drug use & & $\underline{\sim}$ & $\underline{v}$ & $\underline{\sim}$ \\
\hline Statin therapy drug use & & $\underline{v}$ & $\underline{r}$ & $\underline{r}$ \\
\hline Heart rate control drug use & & $\underline{v}$ & $\underline{v}$ & $\underline{\sim}$ \\
\hline Suggested to visit GP & & $\underline{\sim}$ & $\underline{v}$ & $\underline{\square}$ \\
\hline $\begin{array}{l}\text { Visited GP after the } \\
\text { suggestion }\end{array}$ & & & $\underline{v}$ & $\underline{\square}$ \\
\hline
\end{tabular}

$\checkmark$ Intervention delivered 2-3 days after T0 for blood results to be issued

$\checkmark *$ Control delivered 2-3 days after TO for blood results to be issued 


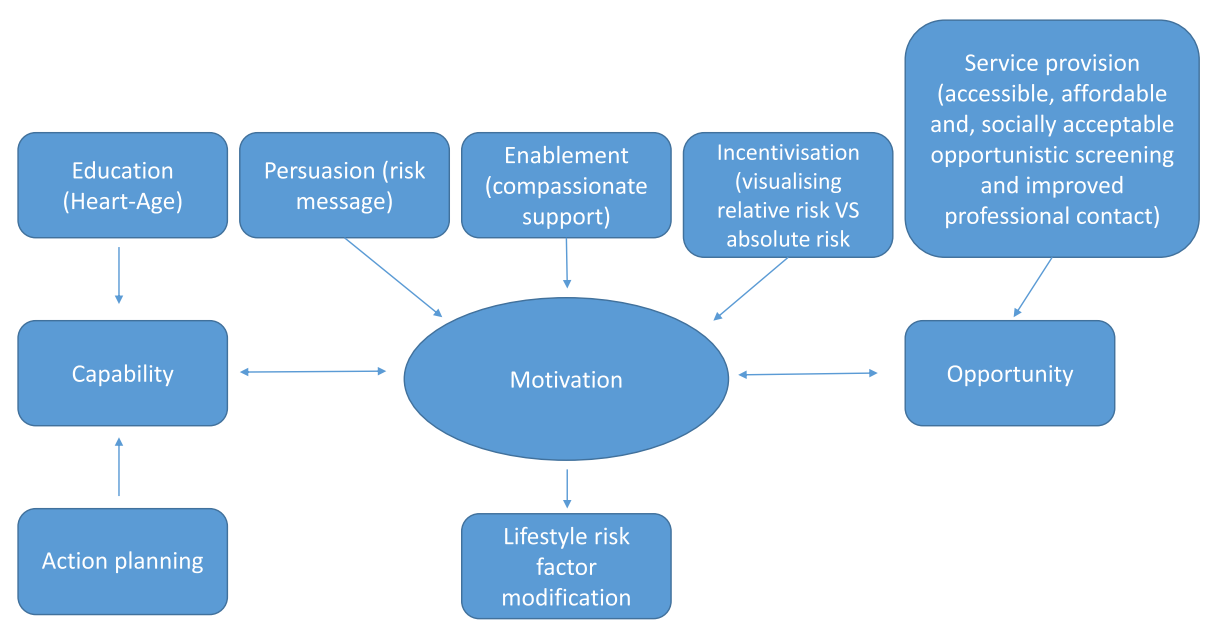

Fig. 3 Application of COM-B in the CRISO program to modify risk

sufficient as individuals still need to understand their risk, develop the skills required to reduce it, and have good access to cardiovascular risk assessment and follow-up. In this way, the interaction between Capability, Opportunity, and Motivation could be pivotal in supporting and bringing about the desired behaviour changes [43]. Figure 3 shows the application of COM-B in the CRISO program to modify risk. This approach was adopted from Michie et al. [53].

\section{Strengths and limitations}

The Intervention's delivery and all measurements will be performed by the same researcher, producing more consistency. A guide was developed to support the fidelity of the Intervention. A randomly chosen set of 10 audio-recorded sessions will be assessed against an intervention guide. A randomised controlled design was selected as it would allow testing for potential intervention effectiveness. This can provide supportive data for preventive program development to be tested on a larger sample future RCT. Part of the study will include conducting interviews, which will help us understand participants' views about the Intervention's implementation and acceptability. This data will allow valuable information on what components are useful to deliver the intervention and support risk factor modification in potential risk groups. The internal pilot phase will dictate any modifications needed in the intervention. However, the data collected for this purpose is proposed in the final efficacy analysis, disregarding that part of the data which came from a pilot phase.

\section{Future research opportunities}

The CRISO study has considered the possibility of future studies. If this study were to establish feasibility, a large scale, randomised controlled trial will be designed and implemented, with the possibility to facilitate the assessment of countrywide effects. This study would be the first in Malta to test the effectiveness of an evidenceinformed intervention to support first-degree relatives to modify risk.

As the study is to recruit first-degree relatives, these would generally have a combination of clinical, biochemical, and genetic risk factors $[54,55]$. In the CRISO study, intervention participants who are found to have alarming parameters (atherogenic lipid profile, obesity) will be asked to consent for blood genetic testing at a 1year time point. Having such samples will open another route for future research to determine if there is an association between genetic risk score (a combination of variants) in those with alarming parameter/s (phenotypestraditional risk factors). If an association is found, this may suggest that conventional risk should be combined with genetic risk, which may give the total potential risk. This study might further refine preventive cardiology.

\section{Counterbalancing for confounding factors and bias}

There are several possible interacting variables: age, health literacy, educational levels, isolation, employment, sex, psychometric scales, time of the cardiac patient event, and family clustering effects. These multiple variables make it rather complex to decide on stratified sampling. Additionally, as the sample solely depends on the recruitment process, an element that still needs to be tested, it was decided not to stratify at this phase.

The chosen method to generate the allocation sequence should produce comparable groups. However, due to a lack of resources, allocation concealment is not possible. This study is single-blinded, where participants will only be informed about the aim of the study but not about being part of an intervention or control group. However, the researcher will be aware of which 
participants have been allocated to the intervention group. Thus, there is a risk of performance and detection bias. An independent person from the study will perform group allocation. Also, group allocation will take place before the baseline risk assessment. Using these methods will avoid any attempt to allocate participants to the Intervention according to their risk profile. This step is necessary when the study's nature does not allow proper blinding [56].

Numbers of the total sample randomised, those lost to follow-up, and reasons for withdrawal will be recorded for each group. The risk of attrition bias will be minimised by carrying forward the last observation. This method will keep the groups balanced if there is a loss to follow-up/missed measuring time points.

\section{Conclusions}

This research will provide new knowledge about the feasibility of the processes and inform the intervention components' acceptability by siblings and offspring. The Intervention may provide a new preventative approach to support individuals to modify their risk behaviours.

\section{Study status}

The study started recruiting in September 2020.

\begin{abstract}
Abbreviations
ASCVD: Atherosclerotic cardiovascular disease; CHD: Coronary heart disease; CVD: Cardiovascular diseases; LDL-C: Low-density lipoprotein cholesterol; MEDAS: Mediterranean diet adherence screener; RAPA: Rapid assessment of physical activity; CDC: Centres for Disease Control and Prevention; HLQ: Health literacy questionnaire; ACS: Acute coronary syndrome; COMB: Capability Opportunity Motivation-Behaviour
\end{abstract}

\section{Acknowledgements}

We gratefully acknowledge the support from the Cardiac Rehabilitation Unit at Mater Dei Hospital toward this research.

\section{Authors' contributions}

JLM: Funding acquisition, Conceptualisation, Methodology, Project administration, Resources, Software, Visualisation, Writing -original draft, and Writing — review and editing. JS: Writing —review and editing. FA: Conceptualisation, Methodology, and Writing—-review and editing. JG: Supervision, Methodology, and Writing - review and editing. The authors read and approved the final manuscript.

\section{Authors' information}

JLM received his B.Sc. (Hons) in Nursing at the University of Malta. He completed his MSc in Preventive Cardiology at Imperial College, London. JLM currently works as an academic and a researcher at the University of Malta.

JS received his PhD from Cardiff University, UK. JS is a Fellow of the UK Royal Statistical Society and is a Chartered Mathematician and currently works as a Senior Lecturer in Biomedical Statistics at the University of Huddersfield, UK. FA received her PhD from Monash University, Melbourne, Australia. FA is a Fellow of the European Society of Cardiology and currently works as a clinical academic Professor of Nursing at the University of Huddersfield and Calderdale and Huddersfield NHS Foundation Trust.

JG received his MD degree from the University of Malta. He completed his Ph.D. at the Department of Cardiovascular Sciences, Sheffield University, UK. JG currently works at the University of Malta and is an Associate Professor.

\section{Funding}

The study was funded by the University of Malta. The funding body had no role in the design of the protocol.

\section{Availability of data and materials}

The anonymised participant datasets generated and/or analysed during the current study will be made available in the University of Malta institutional open access data repository https://www.um.edu.mt/library/oar/.

\section{Declarations}

Ethics approval and consent to participate

The study was approved by the University of Malta Ethics Committee (Ref No: FRECMDS_1819_092) and the Malta Health Ethics Committee (HECO3

(T01/20). Written consent will be obtained.

Consent for publication

Not applicable

\section{Competing interests}

The authors declare that they have no competing interests.

\section{Author details}

${ }^{1}$ Faculty of Health Sciences, University of Malta, Msida, Malta. ${ }^{2}$ Faculty of Medicine and Surgery, University of Malta, Msida, Malta. ${ }^{3}$ School of Human of Health Sciences, University of Huddersfield, Huddersfield, UK. ${ }^{4}$ Research and Development, Huddersfield Royal Infirmary, Acre St, Huddersfield, UK.

Received: 12 January 2021 Accepted: 21 June 2021

Published online: 09 August 2021

\section{References}

1. Marina SMA R, Neil S, Roger B, Michael S, Patel J. Power of the pedigree: The Family History Variable for ASCVD risk stratification: American College of Cardiology; 2021.

2. Chow C, Pell A, Walker A, O'Dowd C, Dominiczak A, Pell J. Families of patients with premature coronary heart disease: an obvious but neglected target for primary prevention. Bmj. 2007;335(7618):481-5.

3. Piepoli MF, Hoes AW, Agewall S, Albus C, Brotons C, Catapano AL, et al. 2016 European Guidelines on cardiovascular disease prevention in clinical practice: The Sixth Joint Task Force of the European Society of Cardiology and Other Societies on Cardiovascular Disease Prevention in Clinical Practice (constituted by representatives of 10 societies and by invited experts) Developed with the special contribution of the European Association for Cardiovascular Prevention \& Rehabilitation (EACPR). Eur Heart J. 2016;37(29): 2315-81.

4. Mifsud JL. The PACl survey: patient adherence to cardioprotective lifestyle interventions in myocardial infarction patients treated with primary percutaneous coronary intervention. Br J Cardiol. 2017;24(4):147.

5. Kolber MR. Family history of cardiovascular disease. J Coll Fam Physicians Can. 2014;60(11):1016.

6. Kral BG, Becker LC, Vaidya D, Yanek LR, Becker DM. Silent myocardial ischaemia and long-term coronary artery disease outcomes in apparently healthy people from families with early-onset ischaemic heart disease. Eur Heart J. 2011:32(22):2766-72.

7. Kral BG, Becker DM, Vaidya D, Yanek LR, Becker LC. Severity of inducible myocardial ischemia predicts incident acute coronary syndromes in asymptomatic individuals with a family history of premature coronary artery disease. J Nucl Cardiol. 2012;19(1):28-36.

8. Ranthe MF, Petersen JA, Bundgaard H, Wohlfahrt J, Melbye M, Boyd HA. A detailed family history of myocardial infarction and risk of myocardial infarction-a nationwide cohort study. PLoS One. 2015;10(5):e0125896.

9. Chow CK, Islam S, Bautista L, Rumboldt Z, Yusufali A, Xie C, et al. Parental history and myocardial infarction risk across the world: the INTERHEART Study. J Am Coll Cardiol. 2011;57(5):619-27.

10. Fischer M, Mayer B, Baessler A, Riegger G, Erdmann J, Hengstenberg C, et al. Familial aggregation of left main coronary artery disease and future risk of coronary events in asymptomatic siblings of affected patients. Eur Heart J. 2007;28(20):2432-7.

11. Soman S, Kar SS, Satheesh S, Ramalingam A. Cardiovascular disease risk profiling among first degree relatives of coronary artery disease patients 
admitted in cardiology ward of JPMER, Puducherry. J Cardiovasc Dis Res. 2016;7(3):108-11.

12. Yanek LR, Moy TF, Blumenthal RS, Raqueno JV, Yook RM, Hill MN, et al. Hypertension among siblings of persons with premature coronary heart disease. Hypertension. 1998;32(1):123-8.

13. Hurrell C, Wietlisbach V, Jotterand V, Volet M, Lenain V, Nicod P, et al. High prevalence of major cardiovascular risk factors in first-degree relatives of individuals with familial premature coronary artery disease-the GENECARD project. Atherosclerosis. 2007;194(1):253-64.

14. Allen JK, Young DR, Blumenthal RS, Moy TF, Yanek LR, Wilder L, et al. Prevalence of hypercholesterolemia among siblings of persons with premature coronary heart disease: application of the Second Adult Treatment Panel guidelines. Arch Intern Med. 1996;156(15):1654-60.

15. Arnett DK, Blumenthal RS, Albert MA, Michos ED, Buroker AB, Miedema MD, et al. 2019 ACC/AHA guideline on the primary prevention of cardiovascular disease. J Am Coll Cardiol. 2019;74:177-232.

16. Perk J, De Backer G, Gohlke H, Graham I, Reiner Ž, Verschuren WM, et al. European Guidelines on cardiovascular disease prevention in clinical practice (version 2012): The Fifth Joint Task Force of the European Society of Cardiology and Other Societies on Cardiovascular Disease Prevention in Clinical Practice (constituted by representatives of nine societies and by invited experts). Atherosclerosis. 2012;223(1):1-68.

17. Critchley JA, Capewell S. Mortality risk reduction associated with smoking cessation in patients with coronary heart disease: a systematic review. JAMA. 2003:290(1):86-97.

18. Pignone M, Phillips C, Mulrow C. Use of lipid lowering drugs for primary prevention of coronary heart disease: meta-analysis of randomised trials. BMJ. 2000;321(7267):983.

19. Trialists'Collaboration A. Collaborative meta-analysis of randomised trials of antiplatelet therapy for prevention of death, myocardial infarction, and stroke in high risk patients. BMJ. 2002;324(7329):71-86.

20. Freemantle N, Cleland J, Young P, Mason J, Harrison J. $\beta$ Blockade after myocardial infarction: systematic review and meta regression analysis. BMJ. 1999;318(7200):1730-7

21. Wen CP, Wai JPM, Tsai MK, Yang YC, Cheng TYD, Lee M-C, et al. Minimum amount of physical activity for reduced mortality and extended life expectancy: a prospective cohort study. Lancet. 2011;378(9798):1244-53.

22. Sofi F, Abbate R, Gensini GF, Casini A. Accruing evidence on benefits of adherence to the Mediterranean diet on health: an updated systematic review and meta-analysis. Am J Clin Nutr. 2010;92(5):1189-96.

23. Estruch R, Ros E, Salas-Salvadó J, Covas M-I, Corella D, Arós F, et al. Primary prevention of cardiovascular disease with a Mediterranean diet. N Engl J Med. 2013:368(14):1279-90.

24. Khera AV, Emdin CA, Drake I, Natarajan P, Bick AG, Cook NR, et al. Genetic risk, adherence to a healthy lifestyle, and coronary disease. N Engl I Med. 2016;375(24):2349-58.

25. Razquin C, Martinez JA, Martinez-Gonzalez MA, Corella D, Santos JM, Marti A. The Mediterranean diet protects against waist circumference enlargement in 12Ala carriers for the PPARY gene: 2 years' follow-up of 774 subjects at high cardiovascular risk. Br J Nutr. 2009;102(5):672-9.

26. Vimaleswaran KS, Li S, Zhao JH, Luan J, Bingham SA, Khaw K-T, et al. Physical activity attenuates the body mass index-increasing influence of genetic variation in the FTO gene. Am J Clin Nutr. 2009;90(2):425-8.

27. Mitchell JA, Church TS, Rankinen T, Earnest CP, Sui X, Blair SN. FTO genotype and the weight loss benefits of moderate intensity exercise. Obesity. 2010; 18(3):641-3.

28. Temelkova-Kurktschiev T, Stefanov T. Lifestyle and genetics in obesity and type 2 diabetes. Exp Clin Endocrinol Diabetes. 2012;120(01):1-6.

29. Mifsud JL, Galea J, Garside J, Stephenson J. Astin F. Motivationa interviewing to support modifiable risk factor change in individuals at increased risk of cardiovascular disease: A systematic review and metaanalysis. PLoS One. 2020;15(11):e0241193.

30. Karmali KN, Persell SD, Perel P, Lloyd-Jones DM, Berendsen MA, Huffman MD. Risk scoring for the primary prevention of cardiovascular disease. Cochrane Database Syst Rev. 2017;3.

31. Riegel B, Moser DK, Buck HG, Dickson W, Dunbar SB, Lee CS, et al. Self-care for the prevention and management of cardiovascular disease and stroke: A scientific statement for healthcare professionals from the American Heart Association. J Am Heart Assoc. 2017;6(9):e006997.

32. Creswell JW, Clark VLP. Designing and conducting mixed methods research. Newbury Park, California: Sage publications; 2017.
33. Martínez-González MA, García-Arellano A, Toledo E, Salas-Salvado J, BuilCosiales P, Corella D, et al. A 14-item Mediterranean diet assessment tool and obesity indexes among high-risk subjects: the PREDIMED trial. PLoS One. 2012;7(8):e43134.

34. Ruiz-Comellas A. How can physical activity be measured in primary health care? Ann Sports Med Res. 2014;1(2):1008.

35. Soureti A, Hurling R, Murray P, van Mechelen W, Cobain M. Evaluation of a cardiovascular disease risk assessment tool for the promotion of healthier lifestyles. Eur J Prev Cardiol. 2010;17(5):519-23.

36. Elsworth GR, Beauchamp A, Osborne RH. Measuring health literacy in community agencies: a Bayesian study of the factor structure and measurement invariance of the health literacy questionnaire (HLQ). BMC Health Serv Res. 2016;16(1):508.

37. Osborne RH, Batterham RW, Elsworth GR, Hawkins M, Buchbinder R. The grounded psychometric development and initial validation of the Health Literacy Questionnaire (HLQ). BMC Public Health. 2013;13(1):1-17.

38. Sullivan GM, Feinn R. Using effect size - or why the P value is not enough. J Grad Med Educ. 2012;4(3):279-82.

39. Lancaster GA, Dodd S, Williamson PR. Design and analysis of pilot studies: recommendations for good practice. J Eval Clin Pract. 2004;10(2):307-12.

40. Hertzog MA. Considerations in determining sample size for pilot studies. Res Nurs Health. 2008:31(2):180-91.

41. Feeley N, Cossette S, Côté J, Héon M, Stremler R, Martorella G, et al. The importance of piloting an RCT intervention. Can J Nurs Res Arch. 2009;15(2): 84-99.

42. Michie S, Richardson M, Johnston M, Abraham C, Francis J, Hardeman W, et al. The behavior change technique taxonomy ( $v 1)$ of 93 hierarchically clustered techniques: building an international consensus for the reporting of behavior change interventions. Ann Behav Med. 2013;46(1):81-95.

43. Atkins $L$, Francis J, Islam R, O'Connor D, Patey A, Ivers N, et al. A guide to using the Theoretical Domains Framework of behaviour change to investigate implementation problems. Implement Sci. 2017;12(1):77.

44. Jackson C, Eliasson L, Barber N, Weinman J. Applying COM-B to medication adherence: a suggested framework for research and interventions. Eur Health Psychol. 2014;16(1):7-17.

45. Barker F, Atkins L, de Lusignan S. Applying the COM-B behaviour model and behaviour change wheel to develop an intervention to improve hearing-aid use in adult auditory rehabilitation. Int J Audiol. 2016;55(sup3):S90-S8.

46. Alexander KE, Brijnath B, Mazza D. Barriers and enablers to delivery of the Healthy Kids Check: an analysis informed by the Theoretical Domains Framework and COM-B model. Implement Sci. 2014;9(1):60.

47. Neuner-Jehle S, Senn O, Wegwarth O, Rosemann T, Steurer J. How do family physicians communicate about cardiovascular risk? Frequencies and determinants of different communication formats. BMC Fam Pract. 2011; 12(1):15.

48. Harris T. Medical statistics made easy: Royal College of General Practitioners; 2014.

49. Braun V, Clarke V. Using thematic analysis in psychology. Qual Res Psychol. 2006;3(2):77-101.

50. Hollis $\mathrm{S}$, Campbell F. What is meant by intention to treat analysis? Survey of published randomised controlled trials. BMJ. 1999:319(7211):670-4.

51. Adams-Huet B, Ahn C. Bridging clinical investigators and statisticians: writing the statistical methodology for a research proposal. J Investig Med. 2009;57(8):818-24

52. Craig P, Dieppe P, Macintyre S, Michie S, Nazareth I, Petticrew M. Developing and evaluating complex interventions: the new Medical Research Council guidance. BMJ. 2008;337:a1655.

53. Michie S, Atkins L, West R. The Behaviour Change Wheel: A Guide to Designing Interventions (Silverback). London; 2014.

54. Natarajan P, O'donnell CJ. Reducing cardiovascular risk using genomic information in the era of precision medicine. Circulation. 2016;133(12):11559.

55. Kashani M, Eliasson A, Vernalis M, Costa L, Terhaar M. Improving assessment of cardiovascular disease risk by using family history: an integrative literature review. J Cardiovasc Nurs. 2013;28(6):E18-27.

56. Bhide A, Shah PS, Acharya G. A simplified guide to randomized controlled trials. Acta Obstet Gynecol Scand. 2018;97(4):380-7.

\section{Publisher's Note}

Springer Nature remains neutral with regard to jurisdictional claims in published maps and institutional affiliations. 